\title{
4.'
}

\section{Beiträge zur näherungsweisen Berechnung bestimmter Integrale nach der Methode der Quadraturen.}

(Yon Herrn Prof. J. L. Raabe zu Zürich,)

Die Methode der Quadraturen zur näherungsweisen Berechnung bestimmter Integrale erhielt erst durch Poissons Bearbeitung *) dieses Gegenstandes eine streng wissenschaftliche Begründung. Denn die, dieser Methode von Maclaurin und Euler beigefügte Correctionsreihe ermangelte eines Kennzeichens aus dem der jedesmal Statt habende Fehler beurtheilt werden könnte. Diese Lücke ist durch das von Poisson dieser Reihe noch beigefügte Ergänzungsglied völlig ausgefüllt, wodurch in theoretischer Beziehung der Gegenstand als geschlossen angesehen werden darf. Für die Anwendung hingegen ist der grofse Vortheil dieses Ergïnzungsgliedes bis jetzt nicht genügend aufgedeckt worden, So ist z. B. der Fall, wenn sämmtliche Glieder der Correctionsreihe in Nullen übergehen, nur oberflächlich erläutert. Das Paradoxe desselben ist zwar durch das Dasein des Ergänzungsgliedes gehoben; allein man sieht nicht recht ab, wie dann nach der Quadraturmethode die Rechnung zu fähren sei. Im Allgemeinen vermifst man ein Verfahren, welches den Genauigkeitsgrad eines nach der Methode der Quadraturen bestimmten Integrals anzeigt, wenn mit irgend einem Iucremente der Variabeln die Rechnung angestellt wird, und umgekehrt.

Die Aufklärung dieser Puncte wird den Inhalt der vorliegenden Beiträge ausmachen.

1. Stellt $\varphi(x)$ eine innerbalb $a$ und $b(b>a)$ continuirliche Function von $x$ dar und setzt man

$$
b-a=n \omega,
$$

wo $n$ eine ganze positive Zahl bedeutet, so enthält eine der folgenden drei Gleichungen:

*) Mémoires de l'institut 1823. 
$\int_{a}^{b} \phi(x) d x=\omega[\phi(a)+\phi(a+\omega)+\phi(a+2 \omega)+\ldots+\phi(a+(n-1) \omega)]$,

$\int_{a}^{b} \phi(x) d x=\omega[\phi(a+\omega)+\phi(a+2 \omega)+\ldots .+\phi(a+(n-1) \omega)+(b)]$,

$\int_{a}^{b} \varphi(x) d x=\omega\left[\frac{1}{2} \phi(a)+\varphi(a+\omega)+\varphi(a+2 \omega)+\ldots .+\phi(a+(n-1) \omega)+\frac{1}{2} \phi(b)\right]$

die Integrationsmethode der Quadraturen. Je kleiner das Increment $\omega$ gedacht wird : mit desto mehr Genauigkeit bestehen auch diese Gleichungen. Diese Gleichungen sind vollkommen richtig, wenn $\omega$ als ein unendlich klein -, also $n$ als ein unendlich grofs - Werdendes gedacht wird. Den Unterschied im Resultate, falls $\omega$ einen endlichen Werth annimmt, welches letztere Resultat nur einen angenäherten Werth darstellt, suchten die Geometer durch Reihen, die nach den Potenzen von $w$ fortgehen, darzustellen. Legendre fand ${ }^{*}$ ), wenn die dritte der obigen Gleichungen festgesetzt wird, diese Reihe unter folgender Form:

$-\boldsymbol{Y}_{2}\left[\varphi_{1}(b)-\emptyset_{1}(a)\right] \omega^{2}+Y_{4}\left[\varphi_{3}(b)-\varphi_{3}(a)\right] \omega^{4}-Y_{6}\left[\Phi_{5}(b)-\varphi_{5}(a)\right] \omega^{6}+\ldots$. wo

$$
\boldsymbol{Y}_{2}=\frac{1}{1.2} B_{1}, \quad \boldsymbol{Y}_{4}=\frac{1}{1.2 .3 .4} B_{2}, \quad \boldsymbol{Y}_{6}=\frac{1}{1.2 .3 .4 .5 .6} B_{6}, \text { u. s. w. }
$$

und $B_{1}, B_{2}, B_{3}$ die Bernouillischen Zahlen sind. Ein Ausdruck von der Form $\varphi_{k}(m)$ stellt hier und in der Folge den $k$ ten Differentialcoëfficienten der Eunction $\varphi(x)$ vor, wenn nach vollzogener Differentiation $x=m$ gesetzt wird.

Die hier aufgestellte Reihe wird die Correctionsreihe zur näherungsweisen Bereehnung der Integrale nach der Quadraturmeţhode genannt.

Endlich hat Poisson, um diese Correctionsreihe sammt ihrem Erzeugungsgliede zu gewinnen, eine mit der folgenden Gleichung ganz ähnliche:

$$
\text { 1. } \begin{aligned}
\int_{a}^{b} \phi(x) d x= & \omega\left[\frac{1}{2} \phi(a)+\varphi(a+\omega)+\varphi(a+2 \omega)+\ldots . .+\varphi(a+(n-1) \omega)+\frac{1}{2} \varphi(b)\right] \\
& -2 \sum_{\rho=1}^{\rho=\infty} \int_{a}^{\dagger} \phi(x) \cos \frac{2 \rho \pi(x-a)}{\omega} d x
\end{aligned}
$$

aufgestellt, in der das Summenzeichen auf alle ganze und positive Werthe von $\rho$ sich erstreckt.

Am schnellsten gelangt man zu derselben, wenn folgende Gleichung: $\varphi(u)=\frac{1}{b-a} \int_{a}^{b} \varphi(x) d x+\frac{2}{b-a} \sum_{r=1}^{r=\infty}\left(\int_{a}^{b} \varphi(x) \cos \frac{r \pi(x-a)}{b-a} d x\right) \cos \frac{r \pi(u-a)}{b-a}$

*) Traité des fonctions elliptiques. T. II. 
zum Grunde gelegt wird, die zur Darstellung der Functionen in Reihen, welche nach den Cosinussen der Vielfachen der Variabeln fortgehen, dient. Wird nun in derselben nach und nach $u=a, u=a+\omega, u=a+2 \omega, \ldots$. $u=a+(n-1) \omega, u=b$ gesetzt und dann die Summe derselben genommen, nachdem man noch früher die erste und die letzte mit $\frac{1}{2}$ multiplicirt hat, so ergiebt sich nach einigen Reductionen die obige Gleichung (1.). Durch Integration p. p. geht das bestimmte Integral in Gleichung (I.):

$$
\int_{a}^{b} \varphi(x) \cos \frac{2 \varrho \pi(x-a)}{\omega} d x
$$

in eine nach aufsteigenden Potenzen von $\omega^{2}$ fortgehende Reibe über, wodurch endlich erhalten wird:

$$
\text { II. } \int_{a}^{b} \varphi(x) d x=
$$

$\omega\left[\frac{1}{2} \varphi(a)+\emptyset(a+\omega)+\varphi(a+2 \omega)+\ldots .+\varphi(a+(n-1) \omega)+\frac{1}{2} \varphi(b)\right]$ $-\boldsymbol{Y}_{2}\left[\varphi_{1}(b)-\varphi_{1}(a)\right] \omega^{2}+\boldsymbol{Y}_{4}\left[\varphi_{3}(b)-\varphi_{3}(a)\right] \omega^{4}-\ldots$

$$
\begin{gathered}
\cdots+(-1)^{m} \boldsymbol{Y}_{2 m}\left[\varphi_{2 m-1}(b)-\varphi_{2 m-1}(a)\right] \omega^{2 m} \\
+2(-1)^{m+1}\left(\frac{\omega}{2 \pi}\right)^{2 m} \sum_{\rho=1}^{\rho=\infty} \frac{1}{\varrho^{2 m}} \int_{a}^{b} \varphi_{2 m}(x) \cdot \cos \frac{2 \varrho \pi(x-a)}{\omega} d x .
\end{gathered}
$$

Diese Gleichung, in welcher:

$$
\text { 1. } Y_{2 k}=\frac{2}{(2 \pi)^{2 k}}\left(1+\frac{1}{2^{2 k}}+\frac{1}{3^{2 k}}+\frac{1}{4^{2 k}}+\ldots .\right)
$$

ist, löset das Problem der näherungsweisen Integration nach der Methode der Quadraturen vollständig.

Die erste Zeile dieser Gleichung drückt die allgemein bekannte Integrationsmethode der Quadraturen aus. Die zweite Zeile corrigirt die erste, falls in derselben $\omega$ endlich vorausgesetzt wird. Die dritte Zeile endlich enthält das Poissonsche Ergänzungsglied, welches zur Schätzung des mit der Correctionsreihe noch begangenen Fehlers dienen soll. Dieses Ergänzungsglied bildet die Basis unserer folgenden Untersuchungen.

2. Stellt man durch $\boldsymbol{R}_{m}$ den numerischen Werth dieses Ergänzungsgliedes dar, 80 ist

und wegen

$$
\boldsymbol{R}_{m}=2\left(\frac{\omega}{2 \pi}\right)^{2 m} \sum_{\rho=1}^{\rho=\infty} \frac{1}{\rho^{2 m}} \int_{a}^{b} \varphi_{2 m}(x) \cos \frac{2 \rho \pi(x-a)}{\omega} d x,
$$

$$
\cos \frac{2 \varrho \pi(x-a)}{\omega}=2\left(\cos \frac{\rho \pi(x-a)}{\omega}\right)^{2}-1
$$

hat man auch 


$$
\begin{aligned}
& \text { 2. } \boldsymbol{R}_{m}=4\left(\frac{\omega}{2 \pi}\right)^{2 m} \sum_{\rho=1}^{\rho=\infty} \frac{1}{\rho^{2 m}} \int_{a}^{b} \varphi_{2 m}(x)\left(\cos \frac{\rho \pi(x-a)}{\omega}\right)^{2} d x \\
& -2\left(\frac{\omega}{2 \pi}\right)^{2 m} \sum_{\rho=1}^{\infty} \frac{1}{\rho^{2 m}} \int_{\alpha}^{b} \varphi_{2 m}(x) d x .
\end{aligned}
$$

Betrachten wir nun zuerst den Fall, wenn die Function $\varphi_{2 m}(x)$ für keinen der Werthe von $x$, die innerhalb $a$ und $b$ liegen, eine Veränderung im Zustande des Zeichens erleidet, oder wenn diese Function von $x=a$ bis $\mid x=b$ dasselbe Zeichen bebält, so besteht, was die numerischen. Werthe betrifft, folgende Ungleichheit:

$$
\begin{aligned}
& \text { 3. } 4\left(\frac{\omega}{2 \pi}\right)^{2 m} \sum_{\rho=1}^{\rho=\infty} \frac{1}{\rho^{2 m}} \int_{a}^{b} \varphi_{2 m}(x)\left(\cos \frac{\rho \pi(x-a)}{\omega}\right)^{2} d x \\
&<4\left(\frac{\omega}{2 \pi}\right)^{2 m} \sum_{\rho=1} \sum_{\rho=\infty} \frac{1}{\rho^{2 m}} \int_{a}^{b} \varphi_{2 m}(x) d x .
\end{aligned}
$$

Daher hat man mit Berücksichtigung des aufgestellten Werthes von $\boldsymbol{R}_{m}$ mit Gleichung (2.)

$$
\boldsymbol{R}_{m}<2\left(\frac{\omega}{2 \pi}\right)^{2 m} \sum_{\varrho=1}^{\varrho=\infty} \frac{1}{\rho^{2 m}} \int_{a}^{b} \phi_{2 m}(x) d x
$$

Nun ist

$$
\int_{a}^{b} \varphi_{2 m}(x) d x=\varphi_{2 m-1}(b)-\varphi_{2 m-1}(a)
$$

Also, mit Zuziehung der Bedeutung von $Y_{2 m}$ nach Gleichung (1.),

$$
\text { 4. } \boldsymbol{R}_{m}<\boldsymbol{Y}_{2 m}\left[\varphi_{2 m-1}(b)-\varphi_{2 m-1}(a)\right] \omega^{2 m} \text {. }
$$

Vergleicht man dieses Ergebnifs mit dem letzten Gliede der Correctionsreihe in Gleichung (II.), so kann man folgenden Satz aufstellen: Wird der Werth eines Integrals nach Gleichung (II.) bestimmt, und bricht mit irgend einem Gliede der Correctionsreike, ж. B. mit dem Gliede

$$
(-1)^{m} \boldsymbol{Y}_{2 m}\left[\varphi_{2 m-1}(b)-\varphi_{2 m-1}(a)\right] \omega^{2 m}
$$

die Rechnung ab, so ist der hiebei begangene Fehler numerisch kleiner, als das letzte noch gerechnete Glied der Correctionsreihe, wenn die Function $\varphi_{2 m}(x)$ von $x=a$ bis $x=b$ ein und dasselbe Zeichen behät.

3. Als Anwendung dieses Theorems eignet sich sehr gut das Integral $\int_{a}^{\infty} e^{-x} \frac{d x}{x}$, wabei $a>0$ gedacht wird.

Es ist hier 


$$
\begin{aligned}
& \varphi(x)=\frac{e^{-x}}{x}, \\
& \varphi_{1}(x)=-\left(\frac{1}{x}+\frac{1}{x^{2}}\right) e^{-x}, 1 \\
& \varphi_{2}(x)=+\left(\frac{1}{x}+\frac{2}{x^{2}}+\frac{2}{x^{3}}\right) e^{-x} \text {, } \\
& \varphi_{3}(x)=-\left(\frac{1}{x}+\frac{3}{x^{2}}+\frac{6}{x^{3}}+\frac{6}{x^{4}}\right) e^{-x} \text {, } \\
& \varphi_{2 m-1}(x)=-\left[x^{2 m-1}+(2 m-1) x^{2 m-2}+(2 m-1)(2 m-2) x^{2 m-3}+\ldots .\right. \\
& \text {.... }+(2 m-1)(2 m-2) \ldots . .4 .3 .2 .1] \frac{e^{-x}}{x^{2 m}} \text {, } \\
& \varphi_{2 m}(x)=+\left[x^{2 m}+2 m x^{2 m-1}+2 m(2 m-1) x^{2 m-2}+\ldots .\right. \\
& \ldots+2 m(2 m-1) \ldots . .4 .3 .2 .1] \frac{e^{-x}}{x^{2 m+1}} \text {. }
\end{aligned}
$$

Dafs die Function $\varphi_{2 m}(x)$ für keinen endlichen Werth von $x$ verschwinden kann, wird aus den positiven Coëfficienten des Ausdruckes innerhalb der Klammern gefolgert; und da dieselbe Function von $x=a$ bis $x=\infty$ continuirlich bleibt, so gelangt man zu dem Schlusse, dafs sie im Bereiche dieser Grenzen beständig positiv bleiben mufs. Wenn daher mit der allgemeinen Gleichung (II.)

$$
\begin{aligned}
\int_{a}^{\infty} e^{-x} \frac{d x}{x} & =\omega\left[\frac{1}{2} \cdot \frac{e^{-a}}{a}+\frac{e^{-(a+\omega)}}{a+\omega}+\frac{e^{-(a+2 \omega)}}{a+2 \omega}+\ldots\right] \\
& -Y_{2}(a+1) e^{-a} \frac{\omega^{2}}{a^{2}}+Y_{4}\left(a^{3}+3 a^{2}+3.2 a+3.2 .1\right) e^{-a} \frac{\omega^{4}}{a^{4}} \ldots \\
& +(-1)^{m} Y_{2 m}\left[a^{2 m-1}+(2 m-1) a^{2 m-2}+(2 m-1)(2 m-2) a^{2 m-3}+\ldots\right. \\
& \ldots . . .(2 m-1)(2 m-2) \ldots 2.1] e^{-a} \frac{\omega^{2 m}}{a^{2 m}}
\end{aligned}
$$

gesetzt und der numerische Werth des Fehlers durch $\boldsymbol{R}_{m}$ angedeutet wird, so besteht folgende Ungleichheit:

$$
\boldsymbol{R}_{m}<1.2 .3 \ldots(2 m-1) \boldsymbol{Y}_{2 m}\left(1+\frac{a}{1}+\frac{a^{2}}{1.2}+\ldots .+\frac{a^{2 m-1}}{1.2 .3 \ldots .(2 m-1)}\right) e^{-a} \cdot \frac{\omega^{2 m}}{a^{2 m}}
$$

Nun ist'

$$
e^{+a}>1+\frac{a}{1}+\frac{a^{2}}{1.2}+\ldots .+\frac{a^{2 m-1}}{1.23 \ldots(2 m-1)}
$$

Daher um so mehr

$$
\boldsymbol{R}_{m}<1.2 .3 \ldots(2 m-1) \boldsymbol{Y}_{2 m} \cdot \frac{\omega^{2 m}}{a^{2 m}}
$$

Setzt man der Kürze wegen

$$
\varepsilon_{m}=1.2 .3 \ldots(2 m-1) Y_{2 m} \frac{\omega^{2 m}}{a^{2 m}},
$$


so ist

Wird $\varepsilon_{m}=\frac{1}{10^{7}}$ angenommen, so findet man

$$
\begin{aligned}
\text { für } \quad m=1, & \frac{\omega}{a}=0^{\prime} 0010954, \\
-m & =2, \quad \frac{\omega}{a}=0^{\prime} 0588566, \\
-m & =3, \quad \frac{\omega}{a}=0^{\prime} 1712248, \\
-\quad m & =4, \quad \frac{\omega}{a}=0^{\prime} 2645613 .
\end{aligned}
$$

Erklären wir uns für den letzten Werth von $\frac{\omega}{a}$, so bestehet die Gleichung

$$
\begin{aligned}
\int_{0}^{\infty} e^{-x} \frac{d x}{x}= & \omega e^{-a}\left[\frac{1}{2 a}+\frac{e^{-\omega}}{a \cdot+\omega}+\frac{e^{-2 \omega}}{a+2 \omega}+\frac{e^{-3 \omega}}{a+3 \omega}+\ldots\right] \\
& -(a+1) Y_{2} \frac{\omega^{2}}{a^{2}} e^{-a}+\left(a^{3}+3 a^{2}+6 a+6\right) Y_{4} \frac{\omega^{4}}{a^{4}} e^{-a} \\
& -\left(a^{5}+5 a^{4}+20 a^{3}+60 a^{2}+120 a+120\right) Y_{6} \frac{\omega^{6}}{a^{6}} e^{-a} \\
& +\left(a^{7}+7 a^{6}+42 a^{5}+210 a^{4}+840 a^{3}+2520 a^{2}+5040 a+5040\right) Y_{8} \frac{\omega^{8}}{a^{8}} e^{-a}
\end{aligned}
$$

mit einer Genauigkeit, die sich noch auf die siebente Decimalstelle erstreckt. Denn das letzte Glied in dieser Gleichung ist nach dem Vorhergehenden numerisch kleiner als $\frac{1}{10^{7}}$, und die Ergänzung der Correctionsreihe ist nach dem aufgestellten Satze kleiner als das letzte Glied derselben. Um Erleichterung im Rechnen zu erzwecken, ohne den Genauigkeitsgrad des Resultats zu verringern, kann man $\frac{\omega}{a}=\frac{1}{4}$ setzen.

Für den ganz speciellen Fall, wenn man $a=1$ hat, erhält man:

$$
\begin{aligned}
\int_{1}^{\infty} e^{-x} \frac{d x}{x}= & \left.\frac{1}{4 e}\left[\frac{1}{2}+\frac{e^{-\frac{1}{4}}}{1+\frac{1}{4}}+\frac{e^{-\frac{2}{4}}}{1+\frac{2}{4}}+\frac{e^{-\frac{3}{3}}}{1+\frac{3}{4}}+\frac{e^{-\frac{4}{4}}}{1+\frac{4}{4}}+\ldots\right]\right] \\
& -\frac{2}{e} Y_{2}\left(\frac{1}{4}\right)^{2}+\frac{16}{e} Y_{4}\left(\frac{1}{4}\right)^{4}-\frac{326}{e} Y_{6}\left(\frac{1}{4}\right)^{6}+\frac{13700}{e} Y_{8}\left(\frac{1}{4}\right)^{8}
\end{aligned}
$$

Die 50 ersten Glieder innerhalb der Klammern der ersten Zeile habe ich berechnet und mit $\frac{1}{4 e}$ die Summe derselben multiplicirt. Als Resultat ergab sich:

$$
\text { 0'22318,50. }
$$

Schơn in der zweiten Decimalstelle weicht dieses Resultat von dem bis jetzt bekannten Werthe des vorliegenden Integrals ab. Nun hat man: 


$$
\begin{array}{lll}
\boldsymbol{Y}_{2}=0^{\prime} 0833333 & \text { und } & \log \boldsymbol{Y}_{2}=0^{\prime} 9208188-2, \\
\boldsymbol{Y}_{4}=0^{\prime} 0013889 & - & \log \boldsymbol{Y}_{4}=0^{\prime} 1426675-3, \\
\boldsymbol{Y}_{6}=0^{\prime} 0000331 & - & \log \boldsymbol{Y}_{6}=0^{\prime} 5194182-5, \\
\boldsymbol{Y}_{8}=0^{\prime} 0000008 & - & \log \boldsymbol{Y}_{8}=0^{\prime} 91^{\prime} 73583-7,
\end{array}
$$

Daher, wenn die 4 Glieder der Correctionsreihe berücksichtigt werden,

$$
\int_{1}^{\infty} e^{-x} \frac{d x}{x}=0^{\prime} 2231850-0^{\prime} 0038018=0^{\prime} 2193839 ;
$$

in welchem Resultate sämmtliche sieben Decimalstellen richtig sind.

4. Gehen wir nun zu dem Falle über, wenn die Function $\oint_{2 m}(x)$ beim Uebergange von $x=a$ bis $x=b$ ein oder mehrere mal das Zeichen verändert. In diesem Falle sind die Schlüsse aus No. 2. nicht zuläfslich; namentlich kann man alsdann die Ungleichheit (2.) picht aufstellen; daher auch die Ungleichheit (4.) nicht mehr gefolgert werden darf. Um diesen schwierigen Fall zu lösen, bedenken wir zuerst, dafs die allgemeine Gleichung (II.) nur unter der Bedingung aus der Greichung (I.) abgeleitet werden konnte, dals die Functionen $\varphi_{1}(x), \varphi_{2}(x), \varphi_{3}(x), \ldots, \varphi_{2 m}(x)$ für Werthe von $x$, die innerhalb der Integrationsgrenzen fallen, nicht unendlich grofs werden. Da demnach die Function $\phi_{2 m}(x)$ beim Uebergange von $x=a$ bis $x=b$ eine oder mehrere Zeichenveränderungen hat, so kann dieses nur daher rühren, dafs sie bei dem erwähnten Uebergange ein oder mehrere mal durch Null geht. Seien nun $\alpha_{1}, \alpha_{2}, \alpha_{2}, \ldots \ldots \alpha_{k}$ reelle, die Function $\varphi_{2 m}(x)$ verschwinden machende Werthe von $x$, welche Abänderungen im Zeichenzustande der Function $\varphi_{2 m}(x)$ bewirken, dergestalt, dafs für jeden noch so kleinen Werth von $\boldsymbol{h}$ die Ausdrücke

$$
\varphi_{2 m}\left(\alpha_{k}-h\right) \text { und } \varphi_{2 m}\left(\alpha_{k}+h\right)
$$

entgegengesetzte Zeichen haben; sọ zerlege man das gegebene Integral in eine Summe von Integralen, deren jedes zwei, der Grölse nach auf einander folgende Buchstaben $\alpha_{1}, \alpha_{2}, \alpha_{3}, \ldots \alpha_{k}$ zu Grenzen hat. Diese Integrale sind sïmmtlich nach der Gleichung (II.) zu behandeln. Auf jedes derselben ist das Theorem in No. 2. anwendbar. Will mah überdies für alle diese Integrale gleich viele Glieder der Correotionsreihe in Anspruch nehmen, so wird für jedes ein eignes Increment bestimmt werden müssen, um einen gemeinschaftlichen Genauigkeitsgrad für alle Integrale zu erzieleo.

In diesem Falle ergiebt sich also folgendes Verfahren. 


\section{Man suche diejenigen Wurzeln der Gleichung}

$$
\varphi_{2 m}(x)=0 \text {, }
$$

die gröfser als $a$ und kleiner als $b$ und die Uebergänge des Zeichenzustandes der Function $\varphi_{2 m}(x)$ sind. Bezeichnet man dieselben, wie vorhin, durch $\alpha_{1} \alpha_{2} \alpha_{3} \ldots \ldots \alpha_{k}$, wo

so setze man

$$
a<\alpha_{1}<\alpha_{2}<\alpha_{3} \ldots .<\alpha_{k}<b,
$$

$$
\begin{gathered}
\int_{a}^{b} \varphi(x) d x= \\
\int_{a}^{\alpha_{1}} \phi(x) d x+\int_{\alpha_{2}}^{\alpha_{z}} \phi(x) d x+\int_{a_{2}}^{\alpha_{3}} \varphi(x) d x+\cdots+\int_{\alpha_{k}}^{b} \varphi(x) d x .
\end{gathered}
$$

Wird ferner

$$
n_{0} \omega_{0}=\alpha_{1}-a, n_{1} \omega_{1}=\alpha_{2}-\alpha_{1}, n_{2} \omega_{2}=\alpha_{3}-\alpha_{2}, \ldots n_{k} \omega_{k}=b-\alpha_{k}
$$

gesetzt, wo $n_{0}, n_{1}, n_{2}, \ldots n_{k}$ ganze positive Zahlen sind, und wird

$$
\int_{a}^{\alpha_{1}} \varphi(x) d x=
$$

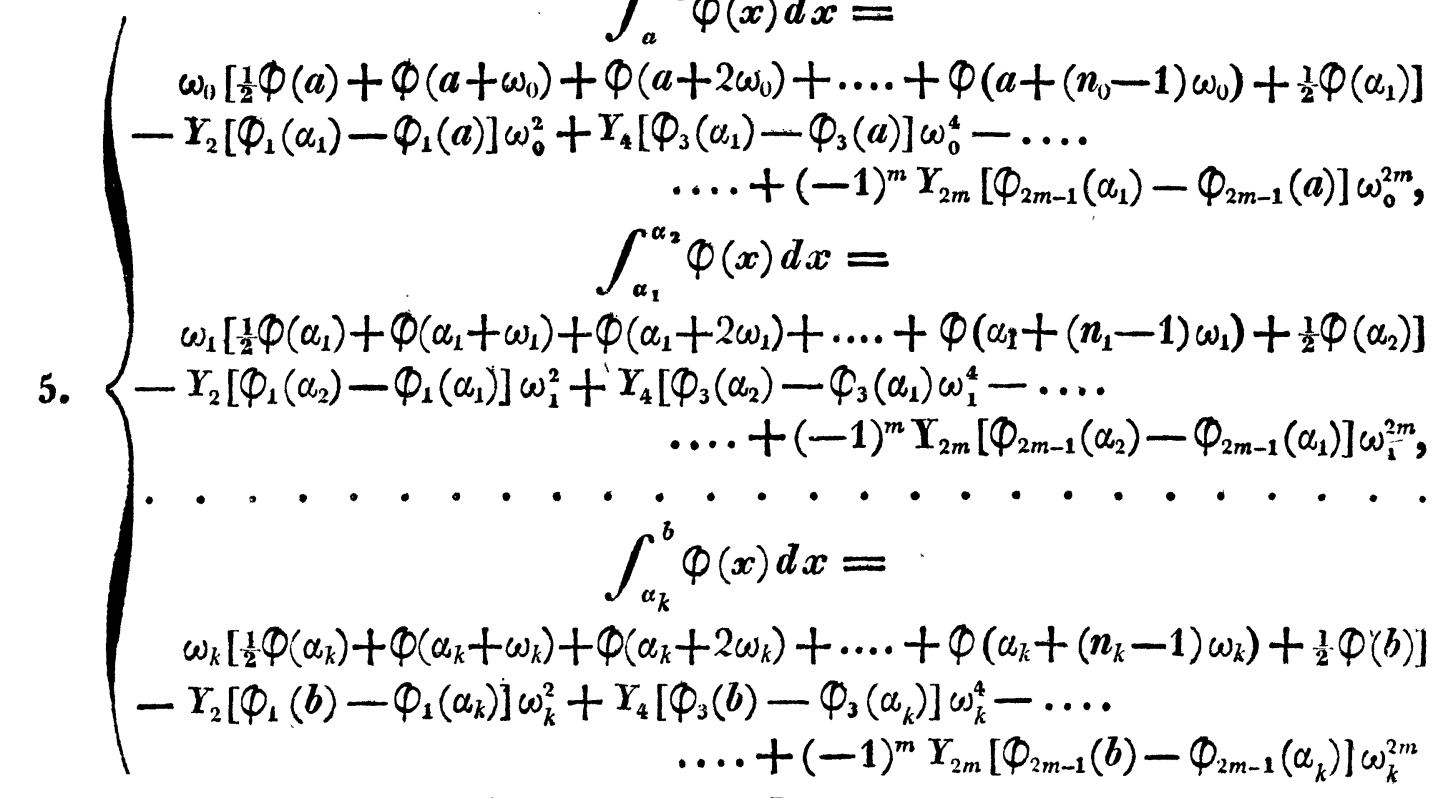
angenommen, so bestimme man die Incremente $\omega_{0}, \omega_{1}, \omega_{2}, \omega_{3}, \ldots \omega_{k}$ aus folgenden Gleichungen:

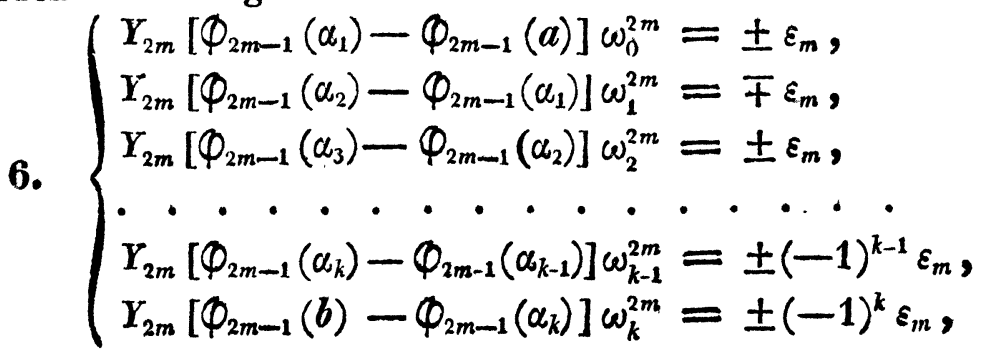


in welchen, damit die Incremente reelle Werthe bekommen, die oberen oder unteren Zeichen gelten, je nachdem die Function $\varphi_{2 m}(x)$ von $x=a$ bis $x=\alpha_{1}$ ein positives ode; negatives Zeichen hat. Wenn mit diesen Incrementen die vorhergehenden Integrale gerechnet werden, so sind die Fehler in den Resultaten überall numerisch kleiner als $\varepsilon_{m}$.

5. Die zuletzt aufgestellten Gleichungen (6.) führen auf einen $\mathbf{Z u}$ sammenhang unter den Incrementen $\omega_{0}, \omega_{1}, \omega_{2}, \ldots \omega_{k}$, der beachtet zu werden verdient. Dividirt man diese Gleichungen, in der Ordnung wie sie aufgestellt sind, durch $\omega_{0}^{2 m}, \omega_{1}^{2 m}, \omega_{2}^{2 m}, \ldots \ldots \omega_{k-1}^{2 m}, \omega_{k}^{2 m}$ und nimmt dann ihre Summe, so stellt die gefundene Gleichung

$$
Y_{2 m}\left[\oint_{2 m-1}(b)-\Phi_{2 m-1}(a)\right]= \pm \varepsilon_{m}\left[\frac{1}{\omega_{0}^{2 m}}-\frac{1}{\omega_{1}^{2 m}}+\frac{1}{\omega_{2}^{2 m}}-\ldots+\frac{(-1)^{k}}{\omega_{k}^{2 m}}\right]
$$

den Zusammenhang unter den Incrementen dar, welcher ganz independent von den Wurzeln $\alpha_{1}, \alpha_{2}, \alpha_{3}, \ldots, \alpha_{k}$ besteht. Der picht selten vorkommende Fall, wenn man

$$
\Phi_{2 m-1}(b)-\varphi_{2 m-1}(a)=0
$$

hat, giebt folgende noch einfachere Relation;

$$
\frac{1}{\omega_{0}^{2 m}}-\frac{1}{\omega_{3}^{2 m}}+\frac{1}{\omega_{2}^{2 m}}-\frac{1}{\omega_{3}^{2 m}}+\ldots+\frac{(-1)^{k}}{\omega_{k}^{2 m}}=0
$$

Für $k=1$ giebt diese Gleichung

$$
\omega_{0}=\omega_{1}
$$

In diesem speciellen Falle wird die Integration von $a$ bis $b$ mit einem einzigen Incremente bewerkstelliget.

Für $k=2$ giebt die Gleichung

$$
\frac{1}{\omega_{i}^{2 m}}=\frac{1}{\omega_{0}^{2 m}}+\frac{1}{\omega_{2}^{2 m}}
$$

woraus gefolgert werden kann, dafs das mittlere Increment, oder dasjenige mit welchem das bestimmte Integral $\int_{\alpha_{1}}^{\alpha_{2}} \phi(x) d x$ ausgemittelt wird, numerisch kleiner als die beiden andern Incremente $\omega_{0}$ und $\omega_{2}$ sein mufs.

6. Die in No. 4. angestellten Betrachtungen heben alle $Z_{w e i f e l}$ wegen dér Benutzung der Correctionsreihe bei der Quadraturmethode. Nur für đie Anwendung wäre es höchst beschwerlich und in vielen Fällen sogar unausführbar, das am gleichen Orte gefolgerte Verfahren zur numerischen Bestimmung eines Integralwerthes auch unverändert befolgen zu müssen. Die folgenden Betrachtungen, welche sich auf die in No. 4. stïtzen, 
führen zu einem viel einfachern Verfahren, welches den Vorzug der Einfachheit mit dem der Gleichförmigkeit in der Behandlung aller Fälle vereint.

Wenn irgend eines der Integrale der Gleichungen (5.) mit dem ihm entsprechenden Incremente aus den Gleichungen (6.) bestimmt wird, so erreicht man jedesmal einen von der Grölse $\varepsilon_{m}$ abhängigen Genauigkeitsgrad. Da dieser Genauigkeitsgrad erhöht werden mufs, wenn nnter übrigens gleichen Verhältnissen ein noch kleineres Increment der Rechnung zum Grunde gelegt wird, so werden viele Schwierigkeiten umgangen, wenn sämmtliche Integrale der Gleichungen (5.) mit dem kleinsten der Incremente $\omega_{0}, \omega_{1}, \omega_{2}, \ldots \omega_{k}$ oder mit einem noch kleinern Incremente bestimmt werden. Nennt man $\omega$ dieses allen Integralen der Gleichungen (5.) zugehörende Increment, so hat man, wenn diese Gleichungen addirt werden und

$$
n \omega=b-a
$$

gesetzt wird, folgende Gleichung:

$$
\text { III. } \int_{a}^{b} \varphi(x) d x=
$$

$\omega\left[\frac{1}{2} \varphi(a)+\varphi(a+\omega)+\phi(a+2 \omega)+\ldots . .+\phi(a+(n-1) \omega)+\frac{1}{2} \varphi(b)\right]$ $-Y_{2}\left[\varphi_{1}(b)-\varphi_{1}(a)\right] \omega^{2}+Y_{4}\left[\varphi_{3}(b)-\varphi_{3}(a)\right] \omega^{4}-\ldots$.

$$
\ldots+(-1)^{m} Y_{2 m}\left[\varphi_{2 m-1}(b)-\emptyset_{2 m-1}(a)\right] \omega^{2 m}
$$

zur Ausmittlung eines bestimmten Integrals.

Dieses Ergebnifs zeigt, dafs man die Quadraturmethode auf gleiche Weise zu corrigiren habe, es möge die Function $\varphi_{2 m}(x)$ von $x=a$ bis $x=\boldsymbol{b}$ beständig ihr Zeichen beibehalten, oder dasselbe mehrere Male ändern. Der Untersobied der beiden Fälle liegt lediglich in der Bestim-

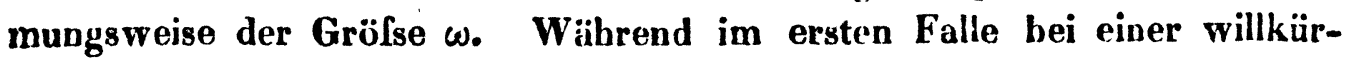
lichen Annahme von $\omega$ der Fehler des Resultats numerisch kleiner als $\varepsilon_{m}$ ist, wo man

$$
\boldsymbol{Y}_{2 m}\left[\varphi_{2 m-1}(b)-\Phi_{2 m-1}(a)\right] \omega^{2 m}= \pm \varepsilon_{m}
$$

hat, mufs im letzten Falle bei der Bestimmung des Increments $\omega$ dio Vorsicht beachtet werden, dafs dasselbe das Minimum unter den Incremente $\omega_{0}, \omega_{1}, \omega_{2}, \ldots . \omega_{k}$ der Gleichungen (6.), oder noch kleiner als dieses Minimum sei, damit ein gleicher Genauigkeitsgrad erzielt werde. Im letztern Falle kann es sich auch ereignen, dafs man

$$
\varphi_{1}(b)-\varphi_{1}(a)=0, \quad \varphi_{3}(b)-\varphi_{3}(a)=0, \ldots . \varphi_{2 m-1}(b)-\varphi_{2 m-1}(a)=0
$$

findet. Dann bat man 


$$
\begin{gathered}
\int_{a}^{b} \varphi(x) d x= \\
. \omega\left[\frac{1}{2} \varphi(a)+\varphi(a+\omega)+\varphi(a+2 \omega)+\ldots .+\varphi(a+(n-1) \omega)+\frac{1}{2} \varphi(b)\right] ;
\end{gathered}
$$

und das nach dieser Gleichung gefundene Resultat differirt von dem unbekannten, wabren Resultate um eine Grörse, die kleiner als $\varepsilon_{m}$ ist, wenn nur $\omega$ die oben erwähnte Eigenschaft des Minimums hat. Das Paradoxon von Legendre ist daher vollständig erläutert; denn das Increment $\omega$ ist nicht völlig willkürlich, sondern es mufs der oben erwähnten Bedingung genïgen.

7. Nach dem eben Vorgetragenen handelt es sich jedesmal um die Ausmittlung des kleinsten der Incremente $\omega_{1}, \omega_{1}, \omega_{2}, \ldots \ldots \omega_{k}, \mathbf{z u}$ welchem Behufe die Kenntnifs dieser sämmtlichen Grölsen unerlafslich ist. Da ferner die Bestimmung dieser Gröfsen eine genaue Kenntnifs der Wurzeln $\alpha_{1}, \alpha_{2}, \alpha_{3}, \ldots \ldots \alpha_{k}$ voraussetzt, und diese $W$ urzeln im Allgemeinen nur angenähert gegeben werden können, so erachten wir es als zweckdienlich, zuerst den Einflufs einer fehlerhaften Annahme der Wurzelwerthe auf die Bestimmung der Incremente zu untersuchen. Wegen der Aehnlichkeit der Gleichungen (6.) unter einander kann man die Untersuchung dadurch erleichtern, dafs man nur ein solches Increment der Betrachtung unterzieht und die für dasselbe gefundenen Resultate, nach gehöriger Umsetzung der Buchstaben, den übrigen Incrementen anpafst.

Legen wir daher die zweite der Gleichungen (6.) zum Grunde und setzen einstweilen

so hat man

$$
\boldsymbol{A}=\sqrt{\frac{ \pm \varepsilon_{m}}{Y_{2 m}}}
$$

$$
\omega_{1}=\boldsymbol{A}\left[\varphi_{2 m-1}\left(\alpha_{2}\right)-\varphi_{2 m-1}\left(\alpha_{1}\right)\right]^{-\frac{1}{2 m}}
$$

Stellt man die genäberten Werthe von $a_{2}$ und $\alpha_{1}$ durch $a_{2}$ und $a_{1}$ vor und den durch diese Annahme erzeugten Werth von $\omega_{1}$ durch $\dot{v}_{1}$, so hat man auch

Nun sei

$$
v_{1}=A\left[\varphi_{2 m-1}\left(a_{2}\right)-\varphi_{2 m-1}\left(a_{1}\right)\right]^{-\frac{1}{2 m}}
$$

$$
a_{2}=\alpha_{2} \pm h_{2}, \quad a_{1}=\alpha_{1} \pm h_{1},
$$

wo also $h_{2}$ und $h_{1}$ die numerischen Werthe der Fehler der. Wurzeln $\alpha_{2}$ und $\alpha_{1}$ vorstellen: so hat man, wenn die dritten und böhern Potenzen dieser Fehler vernachläfsigt und die Gleichungen 


$$
\varphi_{2 m}\left(\alpha_{2}\right)=0, \quad \varphi_{2 m}\left(\alpha_{1}\right)=0
$$

berücksichtigt werden:

$$
v_{1}=\boldsymbol{A}\left\{\varphi_{2 m-1}\left(\alpha_{2}\right)-\varphi_{2 m-1}\left(\alpha_{1}\right)+\frac{1}{2}\left[\varphi_{2 m+1}\left(\alpha_{2}\right) h_{i}^{2}-\varphi_{2 m+1}\left(\alpha_{1}\right) h_{1}^{2}\right]\right\}^{-\frac{1}{2 m}}
$$

Wird nun

$$
\frac{1}{2} \cdot \frac{\varphi_{2 m+1}\left(\alpha_{2}\right)}{\varphi_{2 m-1}\left(\alpha_{2}\right)-\varphi_{2 m-1}^{2}\left(\alpha_{1}\right)}< \pm 1
$$

vorausgesetzt, welche Voraussetzung, der unbestimmten Grölsen $h_{1}$ und $h_{2}$ wegen, immer realisirt werden kann: so erhält man, mit Beachtung des vorhin aufgestellten. Werthes von $\omega_{1}$ :

$$
v_{1}=\omega_{1}\left[1-\frac{1}{4 m} \cdot \frac{\varphi_{2 m+1}\left(\alpha_{2}\right)}{\varphi_{2 m-1}\left(\alpha_{2}\right)-\varphi_{2 m+1}^{2}\left(\alpha_{1}\right)}\right] .
$$

Diese Gleichung stellt den Zusammenhang des Incrementes $\omega_{1}$ mit dessen approximirten Werthe $v_{1}$ dar.

Bezeichnen wir nun durch

$$
a_{1}, a_{2}, a_{3}, a_{4}, \ldots, a_{k}
$$

die genäherten Werthe von $a_{1}, \alpha_{2}, \alpha_{3}, \ldots \alpha_{k}$ und setzen

$$
\boldsymbol{a}_{1}=\alpha_{1} \pm h_{1}, \quad a_{2}=\alpha_{2} \pm h_{2}, \quad a_{3}=\alpha_{3} \pm h_{3}, \ldots . a_{k}=\alpha_{k} \pm h_{k},
$$

so stellen

$$
h_{1}, h_{2} ; h_{3}, h_{4}, \ldots . h_{k}
$$

die numerisohen Werthe der fehlenden Wurzeln $\alpha_{1}, a_{2}, \ldots \alpha_{k}$ dár. Ferner seien

$$
v_{0}, \quad v_{1}, v_{2}, v_{3}, \ldots v_{k}
$$

die genäberten Werthe von $\omega_{0}, \omega_{1}, \omega_{2}, \ldots \omega_{k}$, so hat man, unter Voraussetzung der Ungleichheiten:

$$
\text { 7. }\left\{\begin{array}{c}
\frac{1}{2} \cdot \frac{\varphi_{2 m+1}\left(\alpha_{1}\right) h_{1}^{2}}{\varphi_{2 m-1}\left(\alpha_{1}\right)-\varphi_{2 m-1}(a)}< \pm 1, \\
\frac{\varphi_{2 m+1}\left(\alpha_{2}\right) h_{k}^{2}-\varphi_{2 m+1}\left(\alpha_{1}\right) h_{1}^{2}}{2 \cdot \frac{\varphi_{2 m-1}\left(\alpha_{2}\right)-\varphi_{2 m-1}\left(\alpha_{1}\right)}{\varphi_{2}}}< \pm 1, \\
\cdot \cdot \cdot \cdot \cdot \cdot \cdot \cdot \cdot \cdot \cdot \cdot \cdot \cdot, \\
\frac{\varphi_{2 m+1}\left(\alpha_{k}\right) h_{k}^{2}-\varphi_{2 m+1}\left(\alpha_{k-1}\right) h_{k-1}^{2}}{\varphi_{2 m-1}\left(\alpha_{k}\right)-\varphi_{2 m-1}\left(\alpha_{k-1}\right)}< \pm 1, \\
\frac{1}{2} \cdot \frac{-\varphi_{2 m+1}\left(\alpha_{k}\right) h_{k}^{2}}{\varphi_{2 m-1}(b)-\varphi_{2 m-1}\left(\alpha_{k}\right)}< \pm 1,
\end{array}\right.
$$

und bei Vernachlässigung der dritten und höhern Potenzen von $h_{1}, h_{2}, \ldots$ $\ldots . . h_{k}$, folgende Gleichungen : 


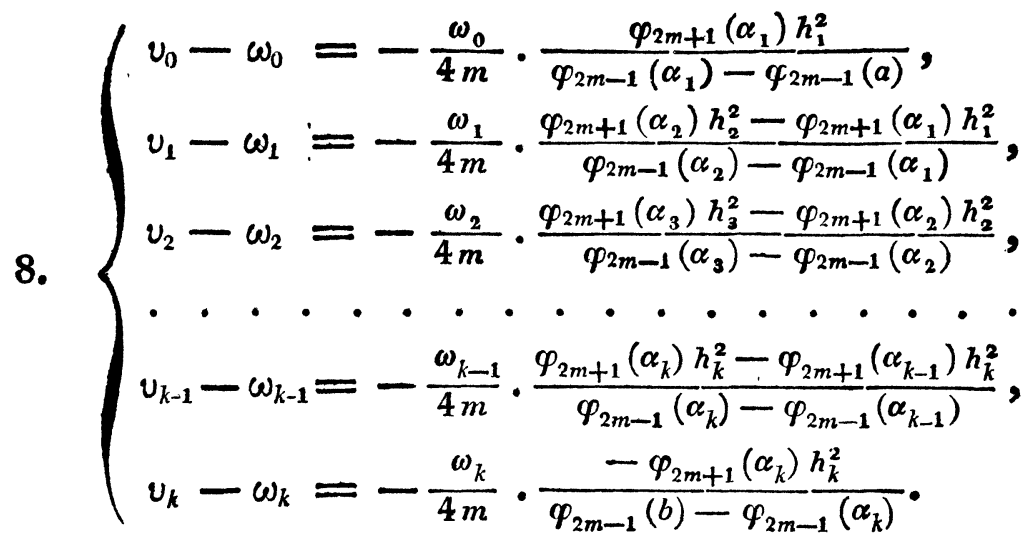

Dafs die Unterschiede $v_{0}-\omega_{0}, v_{1}-\omega_{1}, \ldots$ äufserst kleine Zahlenwerthe haben, leuchtet bei einem einfachen Vergleiche dieser Gleichungen mit den vorhergehenden Ungleichheiten ein; allein eine klare Einsicht in die Beschaffenheit dieser Unterschiede wird erst durch den in der folgenden No. aufgestellten Satz erlangt.

\section{Sämmtliche Differensen}

$$
v_{0}-\omega_{0}, v_{1}-\omega_{1}, v_{2}-\omega_{2}, \ldots v_{k}-\omega_{k}
$$

der Gleichungen (8.) haben positive Werthe.

Wir begründen diesen $S$ sitz folgendermaafsen:

Man setze voraus, die Function $\oint_{2 m}(x)$ habe für alle Werthe von $x=a$ bis $x=\alpha_{2}$ positive, von $x=\alpha_{1}$ bis $x=\alpha_{2}$ negative, von $x=\alpha_{2}$ bis $x=a_{3}$ positive Werthe, u. s. w., so hat dieselbe Function ron $x=\alpha_{k-1}$ bis $x=\alpha_{k}$ Werthe mit dem Zeichen von $(-1)^{k-1}$ und von $x=\alpha_{k}$ bis $x=b$ Werthe mit Zeichen von $(-1)^{k}$. Dieser, oder der genau entgegengesetzte Fall, mufs Statt haben. Lassen wir einstweilen den Gegensatz unbeachtet, so hat man zuerst, wenn unter $\delta$ eine unendlich klein werdende positive Grölse gedacht wird, folgende Gleichungen:

$$
\begin{aligned}
& \varphi_{2 m}\left(\alpha_{1}-\delta\right)=-\delta \varphi_{2 m+1}\left(\alpha_{1}\right) \text { und } \varphi_{2 m}\left(\alpha_{1}+\delta\right)=+\delta \varphi_{2 m+1}\left(\alpha_{1}\right) \text {, } \\
& \varphi_{2 m}\left(a_{2}-\delta\right)=-\delta \varphi_{2 m+1}\left(a_{2}\right) \quad-\quad \varphi_{2 m}\left(a_{2}+\delta\right)=+\delta \emptyset_{2 m+1}\left(a_{2}\right) \text {, } \\
& \varphi_{2 m}\left(a_{3}-\delta\right)=-\delta \varphi_{2 m+1}\left(a_{3}\right)-\varphi_{2 m}\left(a_{3}+\delta\right)=+\delta \varphi_{2 m+1}\left(\alpha_{3}\right) \text {, } \\
& \varphi_{2 m}\left(\alpha_{k}-\delta\right)=-\delta \varphi_{2 m+1}\left(a_{k}\right) \quad-\quad \phi_{2 m}\left(\alpha_{k}+\delta\right)=+\delta \varphi_{2 m+1}\left(\omega_{k}\right)
\end{aligned}
$$

Unter der vorliegenden Annahme zeigt die erste dieser Zeilen für $\varphi_{2 m+1}\left(\alpha_{1}\right)$ ein negatives, die zweite Zeile für $\emptyset_{2 m+1}\left(\alpha_{2}\right)$ ein positives, die dritte Zeile für $\varphi_{2 m+1}\left(a_{3}\right)$ ein negatives Resultat u. s. w. Die letzte Zeile endlich 
zeigt für $\varphi_{2 m+1}\left(\alpha_{k}\right)$ ein Resultat mit dem Zeichen von $(-1)^{k}$. Stellt man daher die Functionen $\varphi_{2 m+1}\left(\alpha_{1}\right), \emptyset_{2 m+1}\left(\alpha_{2}\right), \ldots \varphi_{2 m+1}\left(\alpha_{k}\right)$ in horizontaler Reihe auf, und unter jeder das ihr zugehörende Zeichen, so erbïlt man folgende Anordnung:

$$
\begin{aligned}
& \varphi_{2 m+1}\left(\alpha_{1}\right), \varphi_{2 m+1}\left(\alpha_{2}\right), \varphi_{2 m+1}\left(\alpha_{3}\right), \ldots \varphi_{2 m+1}\left(\alpha_{k-1}\right), \varphi_{2 m+1}\left(\alpha_{k}\right), \\
& \text { - }+\quad-\quad(-1)^{k-1} \quad(-1)^{k} \text {. }
\end{aligned}
$$

Ferner bestehen folgende Gleichungen:

$$
\begin{aligned}
& \varphi_{2 m-1}\left(\alpha_{1}\right)-\varphi_{2 m-1}(a)=\int_{a}^{\alpha_{2}} \varphi_{2 m}(x) d x, \\
& \varphi_{2 m-1}\left(\alpha_{2}\right)-\varphi_{2 m-1}\left(\alpha_{1}\right)=\int_{\alpha_{1}}^{\alpha_{2}} \varphi_{2 m}(x) d x, \\
& \varphi_{2 m-1}\left(\alpha_{3}\right)-\varphi_{2 m-1}\left(\alpha_{2}\right)=\int_{\alpha_{2}}^{\alpha_{2}} \varphi_{2 m}(x) d x, \\
& \cdot \cdot \cdot \cdot \cdot \cdot \cdot \cdot \cdot \cdot \cdot \cdot \cdot \cdot \cdot \cdot \cdot \cdot{ }^{b} \varphi_{2 m}(x) d x .
\end{aligned}
$$

Wird nun der bekannte Zusammenhang zwischen Summe und bestimmtem Integral berücksichtigt, so ergiebt sich folgende Anorduung:

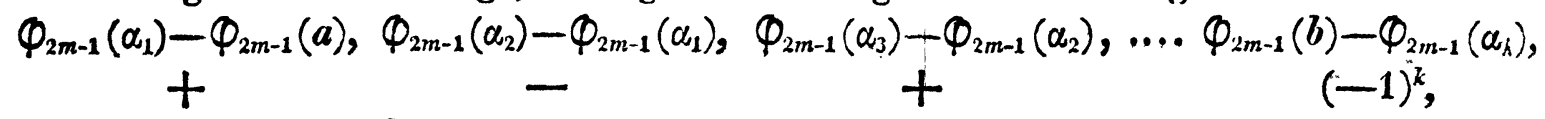

wo jedes Grölsenpaar und das unter demselben stehende Zeichen auf gleiche Weise wie vorhin zusammengehören.

Da man bei der entgegengesetzten Annahme über die Zeichenzustände der Function $\phi_{2 m}(x)$ auf folgende Zusammenstellung der Functionen und deren Zeichen geführt wird:

und

$$
\begin{array}{ccccc}
\varphi_{2 m+1}\left(\alpha_{1}\right), & \varphi_{2 m+1}\left(\alpha_{2}\right), & \varphi_{2 m+1}\left(\alpha_{3}\right), & \ldots & \varphi_{2 m+1}\left(\alpha_{k}\right), \\
+ & - & + & & (-1)^{k+1}
\end{array}
$$

$\varphi_{2 m-1}\left(a_{1}\right)-\varphi_{2 m-1}(a), \varphi_{2 m-1}\left(a_{2}\right)-\varphi_{2 m-1}\left(\alpha_{1}\right), \varphi_{2 m-1}\left(a_{3}\right)-\varphi_{2 m-1}\left(\alpha_{2}\right), \ldots . \varphi_{2 m-1}(b)-\varphi_{2 m-1}\left(a_{k}\right)$, $+$

$(-1)^{k+1}$

so folgt, dafs in den Brüchen der Gleichungen (8.) die Zeichen der Zähler denen der Nenner entgegengesetzt sind; und da vor jedem dieser Brüche das Zeichen - steht, so ist der angekündigte Satz gerechtfertigt.

9. Aus dem im vorigen No, aufgestellten Satze erhält man zuerst folgende Ungleichheiten :
9. $\omega_{0}<v_{0}$,
$\omega_{1}<v_{1}$
$\omega_{2}<v_{2}$,
$w_{k}<v_{k}$, 
oder es sind sämmtliche, durch angenäherte Werthe der Wurzeln $\alpha_{1}, \alpha_{2}, \ldots$ $\ldots \alpha_{k}$ erlangten Incrementenwerthe gröfser als die unbekannten wahren Incrementen werthe.

Ferner folgt aus demselben Satze und mit Zuziehung der Ungleichheiten (7.):

$$
v_{0}-\omega_{0}<\frac{\omega_{0}}{2 m}, \quad v_{1}-\omega_{1}<\frac{\omega_{1}}{2 m}, \quad v_{2}-\omega_{2}<\frac{\omega_{2}}{2 m}, \ldots . v_{k}-\omega_{k}<\frac{\omega_{k}}{2 m},
$$

daher wegen (9.) um so mehr

$$
v_{0}-\omega_{0}<\frac{v_{0}}{2 m}, \quad v_{1}-\omega_{1}<\frac{v_{1}}{2 m}, \quad v_{2}-\omega_{2}<\frac{v_{2}}{2 m}, \quad \ldots \quad v_{k}-\omega_{k}<\frac{v_{k}}{2 m} ;
$$

woraus sich folgende Ungleichheiten ergeben:

$$
\text { 10. } \begin{aligned}
\omega_{0}>v_{0}\left(1-\frac{1}{2 m}\right), \quad \omega_{1}>v_{1}\left(1-\frac{1}{2 m}\right), \omega_{2}>v_{2}\left(1-\frac{1}{2 m}\right), \ldots . \\
\ldots . \omega_{k}>v_{k}\left(1-\frac{1}{2 m}\right) .
\end{aligned}
$$

Diese Ungleichheiten geben die untern Grenzwerthe und die in (9.) aufgestellten zeigen die obern Grenzwerthe der Incremente $\omega_{0}, \omega_{1}, \ldots \omega_{k}$ an. Aus den Unterschieden

$$
\frac{v_{0}}{2 m}, \quad \frac{v_{1}}{2 m}, \quad \frac{v_{2}}{2 m}, \ldots \ldots \frac{v_{k}}{2 m}
$$

dieser äufsersten Grenzwerthe ersieht man am besten, mit weicher Genauigkeit auch angenäherte Werthe von $\alpha_{1}, \alpha_{2}, \ldots \alpha_{k}$ zur Kenntnifs der Incremente führen.

Ferner kann man mit Zuziehung der Ungleichheiten (10.) jedesmal eine Zahl finden, die numerisch kleiner als das kleinste der Incremente $\omega_{0}, \omega_{1}, \omega_{2}, \ldots \omega_{k}$ ist. In der That: es stelle $v_{p}$ die kleinste der Gröfsen $v_{0}, v_{1}, v_{2}, \ldots v_{k}$ vor, so hat man, wenn die ihr unter den Incrementen $\omega_{0}, \omega_{1}, \ldots \omega_{k}$ entsprechende Grölse durch $\omega_{p}$ ausgedrückt wird, nach (10.) folgende Ungleichheit:

$$
\omega_{p}>v_{\rho}\left(1-\frac{1}{2 m}\right)
$$

Ist nun $\omega_{p}$ die kleinste der Größsen $\omega_{0}, \omega_{1}, \omega_{2}, \ldots, \omega_{k}$, so stellt der Ausdruck rechter Hand vom Ungleichheitszeichen den angekündigten Zahlenwerth dar. Findet diese Annahme nicht Statt, sondern ist $\omega_{q}$ das Minimum unter den Incrementen $\omega_{0}, \omega_{1}, \ldots \omega_{k}$, so sei $v_{q}$ der diesem Minimum entsprechende Werth unter den Grölsen $v_{0}, v_{1}, v_{2}, \ldots v_{h}$, Dann wird man zuerst vermöge (10.) haben:

$$
\omega_{q}>v_{q}\left(1-\frac{1}{2 m}\right) \text {. }
$$

Cralke's Journal d. M. Bd. XVIII. Hit. 1. 
Nach der Voraussetzung ist $v_{p}<v_{q}$, daher auch

$$
\omega_{q}>v_{p}\left(1-\frac{1}{2 m}\right) \text {. }
$$

Es ist also, wenn

$$
\text { 11. } \omega=\omega_{p}\left(1-\frac{1}{2 m}\right)
$$

gesetzt wird, diese Grö/se $\omega$ kleiner als das kleinste der Incremente $\omega_{0}$, $\omega_{1}, \omega_{2}, \ldots \omega_{k}$, falls $v_{p}$ die kleinste der durch Rechnung gefundenen Gröfsen $v_{0}, v_{1}, v_{2}, \ldots v_{k}$ ist.

10. Die in der vorhergehenden No. gewonnenen Resultate stützen sich sämmtlich auf das Statthaben der Ungleichheiten (7.). Diese Ungleichheiten bezeichnen nur immer den Genauigkeitsgrad in der Bestimmung der Wurzeln $\alpha_{1}, \alpha_{2}, \alpha_{3}, \ldots \alpha_{k}$, damit jene Resultate auch ibre volle Gültigkeit behalten. Wie die Beurtheilung des Genauigkeitsgrades vorzunehmen sei, erbellet aus den folgenden Betrachtungen. Heben wir eine dieser Ungleichheiten, z. B. die folgende

$$
\frac{1}{2} \cdot \frac{\varphi_{2 m+1}\left(\alpha_{p+1}\right) h_{p+1}^{2}-\varphi_{2 m+1}\left(\alpha_{p}\right) h_{p}^{2}}{\rho_{2 m-1}\left(\alpha_{p+1}\right)-\varphi_{2 m-1}\left(\alpha_{p}\right)}< \pm 1,
$$

heraus, wo $h_{p}$ und $h_{p+1}$ die numerischen Werthe der Fehler der Wurzeln $\alpha_{p}$ und $\alpha_{p+1}$ bedeuten. Nach No. 8. haben die Grölsen $\varphi_{2 m+1}\left(\alpha_{p}\right)$ und $\emptyset_{2 m+1}\left(\alpha_{p+1}\right)$ Resultate mit entgegengesetzten Zeichen: wenn man daher

$$
h_{p}>h_{p+1}
$$

voraussetzt, so wird die vorige Ungleichheit um so eher bestehen, wenn man die folgende

$$
\frac{1}{2} \cdot \frac{\varphi_{2 m+1}\left(\alpha_{p+1}\right)-\varphi_{2 m+1}\left(\alpha_{p}\right)}{\varphi_{2 m-1}\left(\alpha_{p+1}\right)-\varphi_{2 m-1}\left(\alpha_{p}\right)} h_{p}^{2}< \pm 1
$$

zu realisiren sucht. Wird hier

$$
a_{p+1}=a_{p+1} \mp h_{p+1} \quad \text { und } \quad \alpha_{p}=a_{p} \mp h_{p}
$$

gesetzt, wo $a_{p+1}$ und $a_{p}$ die angenäherten Werthe von $\alpha_{p+1}$ und $\alpha_{p}$ sind, so hat man, wenn die dritten Dimensionen der Febler vernachläfsigt werden:

$$
\frac{1}{2} \cdot \frac{\varphi_{2 m+1}\left(a_{p+1}\right)-\varphi_{2 m+1}\left(a_{p}\right)}{\varphi_{2 m-1}\left(a_{p+1}\right)-\varphi_{2 m-1}\left(a_{p}\right)} h_{p}^{2}< \pm 1 .
$$

Hieraus zieht man zur Beurtheilung des gröfsern der Fehler $h_{p}$ und $h_{p+1}$ folgende Ungleichheit:

$$
h_{p}^{*}<4 \cdot\left[\frac{\varphi_{2 m-1}\left(a_{p+1}\right)-\varphi_{2 m-1}\left(a_{p}\right)}{\varphi_{2 m+1}\left(a_{p+1}\right)-\pi_{2 m+1}\left(a_{p}\right)}\right]^{2} .
$$

Die Grörsen $a_{p}$ und $a_{p+1}$ sind immer bekannt; die Werthe von $h_{p}$ und $h_{p+\varepsilon}$ lassen sich immer sohätzen; findet daher die letzte Ungleichheit Statt, so 
kann man zur Bestimmung der Gröfse $v_{p}$ schreiten; im entgegengesetzten Falle mufs man die Fehler $h_{p}$ und $h_{p+1}$ verringern, oder den Genauigkeitsgrad in der Bestimmung der Wurzeln $\alpha_{p}$ und $\alpha_{p+1}$ zu erhöhen suchen.

Hat man sich für alle Werthe von $p=1$ bis $p=k-1$ von der Richtigkeit dieser Ungleichheit überzeugt, so kann man zur Berechnung der Gröfsen $v_{1}, v_{2}, v_{3}, \ldots v_{k-1}$ übergehen. Ferner hat man, ehe zur Berechnung von $v_{0}$ geschritten wird, die Richtigkeit folgender Ungleichheit zu untersuchen:

$$
h_{1}^{4}<4 \cdot\left[\frac{\varphi_{2 m-1}\left(a_{1}\right)-\varphi_{2 m-1}(a)}{\varphi_{2 m+1}\left(a_{1}\right)}\right]^{2},
$$

und endlich, ehe man zur Berechnung von $v_{k}$ übergeht, muls man folgende Ungleichbeit

berzustellen suchen.

$$
h_{k}^{4}<4 \cdot\left[\frac{\varphi_{2 m-1}(b)-\varphi_{2 m-1}\left(a_{k}\right)}{\varphi_{2 m+1}\left(a_{k}\right)}\right]^{2}
$$

11. Zum Schlusse wollen wir eine gedrängte Zusammenstellung aller gewonnenen Resultate geben.

Zuerst hat man nach Gleichung III.

$$
\text { 1. } \int_{a}^{b} \varphi(x) d x=
$$

$\omega\left[\frac{1}{2} \phi(a)+\phi(a+\omega)+\phi(a+2 \omega)+\ldots .+\phi(a+(n-1) \omega)+\frac{1}{2} \phi(b)\right]$ $-\mathbf{Y}_{2}\left[\varphi_{1}(b)-\varphi_{1}(a)\right] \omega^{2}+Y_{4}\left[\varphi_{3}(b)-\varphi_{3}(a)\right] \omega^{4}+\ldots$.

wo $n$ eine ganze positive $Z$ ahl und

$$
\cdots+(-1)^{m} \boldsymbol{Y}_{2 m}\left[\varphi_{2 m-1}(b)-\varphi_{2 m-1}(a)\right] \omega^{2 m} \text {, }
$$

$$
\text { 2. } n \omega=b-a
$$

ist. Um die Grölse $\omega$ anzugeben, damit der nach der obigen Gleichnng bestimmte Werth des Integrals nur noch einen Fehler gestatte, der kleiner als eine gegebene Grölse $\varepsilon_{m}$ ist, untersuche man, ob die Gleichung

$$
\text { 3. } \varphi_{2 m}(x)=0
$$

reelle, innerhalb $a$ und $b$ fallende Wurzeln habe, oder nicht.

Im letztern Falle setze man

$$
\text { 4. } \quad Y_{2 m}^{2}\left[\varphi_{2 m-1}(\boldsymbol{b})-\varphi_{2 m-1}(\boldsymbol{a})\right]^{2} \omega^{4 m}=\varepsilon_{n e}^{2},
$$

so ist der aus dieser Gleichung gefolgerte positive Werth von $\omega$ der gesuchte.

Im erstern Falle bezeichne man die angenäherten Werthe der Wurzeln der Gleichung (3.) durch

so dals

$$
a_{1}, a_{2}, a_{3}, \ldots \ldots a_{k},
$$

$$
a<a_{1}<a_{2}<a_{3} \ldots<a_{k}<b \text {. }
$$


Die numerischen Werthe der Fehler dieser Wurzeln seien in derselben Ordmung durch

$$
h_{1}, h_{2}, h_{3}, \ldots \ldots h_{k}
$$

vorgestellt. Diese angenäherten Wurzelwerthe, sammt den zugehörenden Fehlern, müssen folgenden Bedingungen genügen :

$$
\text { 5. }\left\{\begin{array}{r}
h_{1}^{4}<4 \cdot\left[\frac{\varphi_{2 m-1}\left(a_{1}\right)-\varphi_{2 m-1}(a)}{\varphi_{2 m+1}\left(a_{1}\right)}\right]^{2}, \\
h_{2}^{4} \text { oder } h_{1}^{4}<4 \cdot\left[\frac{\varphi_{2 m-1}\left(a_{2}\right)-\varphi_{2 m-1}\left(a_{1}\right)}{\varphi_{2 m+1}\left(a_{2}\right)-\varphi_{2 m+1}\left(a_{1}\right)}\right]^{2}, \\
h_{3}^{4} \text { oder } h_{2}^{4}<4 \cdot\left[\frac{\varphi_{2 m-1}\left(a_{3}\right)-\varphi_{2 m-1}\left(a_{2}\right)}{\varphi_{2 m+1}\left(a_{3}\right)-\varphi_{2 m+1}\left(a_{2}\right)}\right]^{2}, \\
\left.\cdot \cdot \cdot \cdot \cdot \cdot \cdot \cdot \cdot \cdot \cdot \cdot \cdot \cdot \cdot \cdot \cdot \cdot \cdot \cdot \cdot \frac{\varphi_{2 m-1}\left(a_{k}\right)-\varphi_{2 m-1}\left(a_{k-1}\right)}{\varphi_{2 m+1}\left(a_{k}\right)-\varphi_{2 m+1}\left(a_{k-1}\right)}\right]^{2}, \\
h_{k}^{4} \text { oder } h_{k-1}^{4}<4 \cdot\left[\frac{\varphi_{2 m-1}(b)-\varphi_{2 m-1}\left(a_{k}\right)}{\varphi_{2 m+1}\left(a_{k}\right)}\right]^{2} .
\end{array}\right.
$$

In den Ungleichheiten, wo zwei Nachbarsfehler vorkommen, ist jedesmal der numerisch grölsere zu nehmen. Hat man diesen Bedingungen Genüge gethan, so schreite man zur Bestimmung der Grö́sen

aus folgenden Gleichungen:

$$
v_{0}, \quad v_{1}, v_{2}, \ldots v_{k}
$$

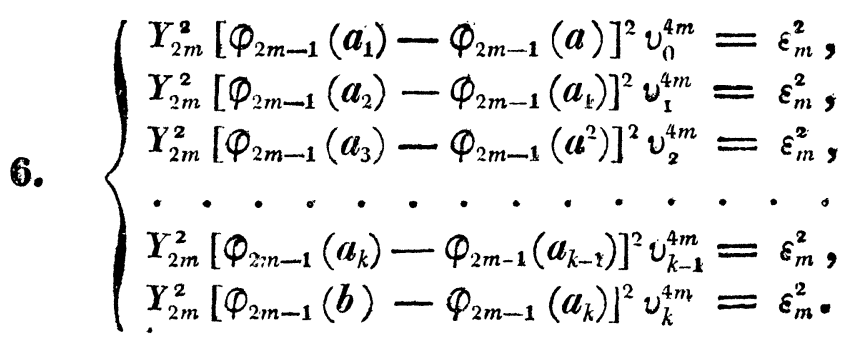

Ist $v_{p}$ die kleinste dieser Grölsen, so ist, wenn

$$
\text { 7. } \omega=v_{p}\left(1-\frac{1}{2 m}\right)
$$

gesetzt wírd, die Grölse $\omega$ die verlangte.

In der folgenden No. werden wir bei der Behandlung einiger speciellen Fälle jedesmal auf die in dieser No. aufgestellten und eigens bezeichneten Resultate hinweisen. Man hat also

12. I. Es sei das Integral $\int_{0}^{\infty} e^{-x^{2}} d x$ zur Berechnung vorgelegt. 


$$
\begin{aligned}
& \varphi(x)=e^{-x^{2}}, \\
& \varphi_{1}(x)=-2 x e^{-x^{2}}, \\
& \varphi_{2}(x)=+2\left(2 x^{2}-1\right) e^{-x^{2}}, \\
& \varphi_{3}(x)=-4 x\left(2 x^{2}-3\right) e^{-x^{2}}, \\
& \varphi_{4}(x)=+4\left(4 x^{4}-12 x^{2}+3\right) e^{-x^{2}}, \\
& \varphi_{5}(x)=-8 x\left(4 x^{4}-20 x^{2}+15\right) e^{-x^{2}}, \\
& \varphi_{6}(x)=+8\left(8 x^{6}-60 x^{4}+90 x^{2}-15\right) e^{-x^{2}}, \\
& \varphi_{7}(x)=-16 x\left(8 x^{6}-84 x^{4}+210 x^{2}-105\right) e^{-x^{2}}, \\
& \varphi_{3}(x)=+16\left(16 x^{9}-224 x^{6}+840 x^{4}-840 x^{2}+105\right) e^{-x^{3}}, \\
& \varphi_{9}(x)=-32 x\left(16 x^{8}-288 x^{6}+1512 x^{4}-2520 x^{2}+945\right) e^{-x^{2}} . \\
& \text { u. s. w }
\end{aligned}
$$

Will man in der Gleichung (1.) mit dem Gliede, welches dem Zeiger $m=3$ entspricht, die Rechnung abbrechen, so suche man nach der Gleichung (3.) die positiven Wurzeln der Gleichung

$$
\varphi_{8}(x)=0
$$

auf. Diese Wurzeln können im gegenwärtigen Falle nur in der folgenden Gleichung rorkommen :

$$
\text { a. } 16 x^{8}-224 x^{6}+840 x^{4}-840 x^{2}+105=0 .
$$

Setzt man hier $2 x^{2}=y$, so hat man

$$
y^{4}-28 y^{3}+210 y^{2}-420 y+105=0 .
$$

Diese Gleichung hat die vier $W$ urzeln reell und positiv. Dieselben sind zwischen 0 und 1 , zwischen 2 und 3 , zwischen 7 und 8 , und endlich zwisehen 17 und 18 enthalten. Bestimmt man die zwischen 0 und 1 liegende Wurzel etwas näher, so überzengt man sich, dafs dieselbe zwischen 0 und $\frac{1}{2}$ enthalten sei. Es liegen somit die positiven $W$ urzeln der vorgelegten Gleichung zwischen 0 und $\frac{1}{2}$, zwischen 1 und $1^{\prime} 224 \ldots$, zwischen $1^{\prime} 8 \% 0 \ldots$ und 2, und endlich zwischen $2^{\prime} 915 \ldots$ und 3 . Setzen wir demnach

$$
\text { b. } \quad a_{1}={ }_{10}^{3}, \quad a_{2}=1, \quad a_{3}=2, \quad a_{t}=3 \text {, }
$$

wo also $a_{1}, a_{2}, a_{3}, a_{i}$ angenäherte Werthe der Wurzeln der vorgelegten Gleichung (a.) sind, so hat man

$$
\text { c. } \quad h_{1}<{ }_{10}^{3}, \quad h_{2}<{ }_{100 \sigma}^{224}, \quad h_{3}<{ }_{1000}^{130}, \quad h_{4}>_{\text {1000 }}^{85} \text {, }
$$

wo $h_{1}, h_{2}, h_{3}, h_{4}$ die Fehler dieser angenïherten Werthe sind.

Setzt man diese $W$ erthe von $a_{1}, a_{2}, a_{3}, a_{4}$ in die Ungleichheiten (5.), so bat man: 


$$
\begin{aligned}
& h_{1}^{2}<\frac{86^{\prime} 774568}{730^{\prime} 23829776}
\end{aligned}
$$

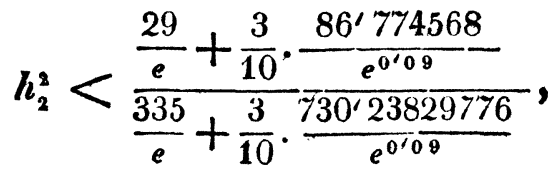

$$
\begin{aligned}
& h_{3}^{2}<\frac{2 \cdot \frac{97}{e^{4}}+\frac{29}{e}}{2 \cdot \frac{721}{e^{4}}+\frac{335}{e}}, \\
& h_{4}^{2}<\frac{813}{4239}
\end{aligned}
$$

oder man muls

$$
\begin{array}{lll}
h_{1}^{2}<0^{\prime} 118 \ldots . . & \text { oder } & h_{1}<0^{\prime} 34 \ldots \\
h_{2}^{2}<0^{\prime} 106 \ldots & - & h_{2}<0^{\prime} 32 \ldots \\
h_{3}^{2}<0^{\prime} 095 \ldots . & \text { - } & h_{3}<0^{\prime} 30 \ldots \\
h_{4}^{2}<0^{\prime} 191 \ldots . . & - & h_{4}<0^{\prime} 43 \ldots .
\end{array}
$$

haben. Nun finden diese Ungleichheiten nach (c) Statt; Jaher kann man mit den obigen Werthen von $a_{1}, a_{2}, a_{3}, a_{4}$ die Rechnung beginnen. Setzt man also in $(b.) \varepsilon_{m}=\frac{1}{10^{7}}$ und berücksichtigt den in No. 3. angeführten Werth von $Y_{\mathrm{s}}$, so hat man, da $m=4$ ist, wegen

$$
\begin{array}{ll}
\varphi_{7}\left(a_{1}\right)=380^{\prime} 669, & \varphi_{7}\left(a_{2}\right)=-170^{\prime} 696, \\
\varphi_{7}\left(a_{3}\right)=+56^{\prime} 852, & \varphi_{7}^{\prime}\left(a_{4}\right)=-4^{\prime} 819
\end{array}
$$

und wegen

$$
\varphi_{7}(a)=0, \quad \varphi_{7}(b)=0
$$

folgende Werthe für $v_{1}, v_{2}, v_{3}, v_{4}$ :

$$
\begin{aligned}
& v_{0}=0^{\prime} 365394 \ldots \\
& v_{1}=0^{\prime} 348859 \ldots \\
& v_{2}=0^{\prime} 389669 \ldots \\
& v_{3}=0^{\prime} 458744 \ldots \\
& v_{4}=0^{\prime} 630950 \ldots
\end{aligned}
$$

Da $v_{1}$ den kleinsten Werth hat, so ist nach (\%.)

$$
\omega=v_{1}\left(1-\frac{1}{8}\right)=0^{\prime} 305251 \ldots
$$

Erklären wir uns für $\omega=\frac{3}{10}$, so hat man nach (1.)

$$
\int_{0}^{\infty} e^{-x^{2}} d x=\omega\left(\frac{1}{2}+e^{-\omega^{2}}+e^{-(2 \omega)^{2}}+e^{-(3 \omega)^{2}}+e^{-(4 \omega)^{2}}+\ldots\right) \text {. }
$$

Sämmtliche Glieder der Correctionsreihe gehen hier in Nullen über. Die Reihe innerhalb der Klammern dieser Gleichung ist so lange fortzusetzen, bis man auf Glieder, kleiner als $\varepsilon_{m}$ oder $\frac{1}{10^{7}}$, stölst.

Mit Zuziehung einer siebenstelligen Logarithmentafel findet man wegen $\log e=0^{\prime} 43429448 \ldots$ folgende Resultate: 


$$
\begin{array}{rlrl}
\frac{1}{2} & =0^{\prime} 5000000, & e^{-(7 \omega)^{2}}=0^{\prime} 0121552, \\
e^{-\omega^{2}}=0^{\prime} 9139310, & e^{-(8 \omega)^{2}}=0^{\prime} 0031511, \\
e^{-(2 \omega)^{2}}=0^{\prime} 6976763, & e^{-(9 \omega)^{2}}=0^{\prime} 0006823, \\
e^{-(3 \omega)^{2}}=0^{\prime} 4448581, & e^{-(10 \omega)^{2}}=0^{\prime} 0001234, \\
e^{-(4 \omega)^{2}}=0^{\prime} 2369277, & e^{-(11 \omega)^{2}}=0^{\prime} 0000186, \\
e^{-(3 \omega)^{2}}=0^{\prime} 1053992, & e^{-(12 \omega)^{2}}=0^{\prime} 0000024, \\
e^{-(6 \omega)^{2}}=0^{\prime} 0391639, & e^{-(13 \omega)^{2}}=0^{\prime} 0000002 .
\end{array}
$$

Addirt man die Zahlen rechter Hand der Gleichheitszeichen, so ergiebt sich : 2'9540894.

Diesen Zablen-Ausdruck mit $\omega=\frac{3}{1^{0}}$ multiplicirt, giebt

$$
\int_{0}^{\infty} e^{-x^{2}} d x=0^{\prime} 88622682 \ldots
$$

Bekanntlich ist der Werth dieses bestimmten Integrals $\frac{1}{2} \sqrt{\pi}$, und wenn dieser Ausdruck in Zah̆len gegeben wird, hat man

$$
\frac{1}{2} \sqrt{\pi}=0^{\prime} 88622692 \ldots
$$

Es differiren die zwei Werthe um $\frac{1}{10^{7}}=\varepsilon_{m}$. Diese Abweichung kann jedoch nur von der Unsicherheit herrühren, mit der ma": die siebente Decimalstelle in einer siebenstelligen Logarithmentafel findet.

11. Als zweites Beispiel legen wir uns das Integral $\int_{0}^{i} e^{-\left(x+\frac{1}{a}\right)} d x$ zur Berechnung vor.

Es ist hier

$$
\begin{aligned}
& \varphi(x)=e^{-\left(x+\frac{1}{x}\right)}, \\
& \varphi_{1}(x)=-\left(1-\frac{1}{x^{2}}\right) e^{-\left(x+\frac{1}{x}\right)} \\
& \varphi_{2}(x)=+\left(1-\frac{2}{x^{2}}-\frac{2}{x^{3}}+\frac{1}{x^{4}}\right) e^{-\left(x+\frac{1}{x}\right)}, \\
& \varphi_{3}(x)=-\left(1-\frac{3}{x^{2}}-\frac{6}{x^{3}}-\frac{3}{x^{4}}+\frac{6}{x^{5}}-\frac{1}{x^{6}}\right) e^{-\left(x+\frac{1}{x}\right)}, \\
& \varphi_{4}(x)=+\left(1-\frac{4}{x^{2}}-\frac{12}{x^{3}}-\frac{18}{x^{4}}+\frac{32}{x^{6}}-\frac{12}{x^{7}}+\frac{1}{x^{8}}\right) e^{-\left(x+\frac{1}{x}\right)}, \\
& \varphi_{5}(x)=-\left(1-\frac{5}{x^{2}}-\frac{20}{x^{3}}-\frac{50}{x^{4}}-\frac{60}{x^{6}}+\frac{50}{x^{6}}+\frac{180}{x^{7}}-\frac{115}{x^{8}}+\frac{20}{x^{9}}\right.
\end{aligned}
$$




$$
\begin{aligned}
& \varphi_{6}(x)=+\left(1-\frac{6}{x^{7}}-\frac{30}{x^{3}}-\frac{105}{x^{4}}-\frac{240}{x^{5}}+\frac{200}{x^{6}}+\frac{540}{x^{7}}-\frac{1095}{x^{8}}-\frac{1080}{x^{9}}\right. \\
& \left.+\frac{294}{x^{10}}-\frac{30}{x^{11}}+\frac{1}{x^{12}}\right) e^{-\left(x+\frac{1}{x}\right)} \\
& \varphi_{7}(x)=-\left(1-\frac{7}{x^{2}}-\frac{42}{x^{3}}-\frac{189}{x^{4}}-\frac{630}{x^{5}}-\frac{1295}{x^{6}}-\frac{420}{x^{7}}+\frac{5075}{x^{8}}+\frac{7140}{x^{8}}\right. \\
& \left.-\frac{10521}{x^{10}}+\frac{3990}{x^{12}}-\frac{623}{x^{12}}+\frac{42}{x^{13}}-\frac{1}{x^{14}}\right) e^{-\left(x+\frac{1}{x}\right)}
\end{aligned}
$$

u. 8. w.

Will man in der Gleichung (1.) mit dem Gliede, dessen Zeiger $m=3$ ist, schliefsen, so suche man die zwischen 0 und 1 enthaltenen positiven Wurzeln der Gleichung

$$
\varphi_{6}(x)=0
$$

auf. Diese Wurzeln können nur in folgender Gleichung vorkommen;

$$
\begin{gathered}
a^{\prime} .0=1-\frac{6}{x^{2}}-\frac{30}{x^{3}}-\frac{105}{x^{4}}-\frac{240}{x^{5}}-\frac{200}{x^{6}}+\frac{540}{x^{7}}+\frac{1095}{x^{8}}-\frac{1080}{x^{9}} \\
+\frac{294}{x^{10}}-\frac{30}{x^{11}}+\frac{1}{x^{12}} .
\end{gathered}
$$

Setzt man hierin $x=\frac{1}{y}$, so ist zu untersuchen, ob die Gleichung

$$
\begin{gathered}
y^{.12}-30 y^{11}+294 y^{10}-1080 y^{9}+1095 y^{8}+540 y^{7}-200 y^{6}-240 y^{.5} \\
-105 y^{4}-30 y^{3}-6 y^{2}+1=0
\end{gathered}
$$

positive Wurzeln besitze, welche die Einheit übertreffen.

Man überzeugt sich nach dem Fourierschen Theorem $\left(A_{.}\right)$, dals die Gleichung nur 4 solche Wurzeln haben kann. Man findet dieselben auf gewöhnlichem Wege, zwischen 2 und 4 , zwischen 4 und 5, zwischen 8 und 9, und zwischen 14 und 15 enthalten. Es liegen somit die verlangten Wurzeln der Gleichung $\left(a^{\prime}\right.$.) 'zwisohen $\frac{1}{2}$ und $\frac{1}{3}$, zwischen $\frac{1}{4}$ und $\frac{1}{5}$, zwischen $\frac{1}{8}$ und $\frac{1}{6}$, und zwischen $\frac{1}{14}$ und $\frac{1}{15}$. Setzt man also

$$
b^{\prime} . \quad a_{1}={ }_{100}^{6}, \quad a_{2}={ }_{10}^{1}, \quad a_{3}=\frac{2}{10}, \quad a_{4}=\frac{4}{10},
$$

so hat man

$$
c^{\prime} . \quad h_{1}<\frac{1}{100}, \quad h_{2}<\frac{1400}{1000}, h_{3}<_{\frac{5}{100}}, \quad h_{4}<\frac{1}{10} .
$$

Werden diese Werthe ron $a_{1}, a_{2}, a_{3}, a_{4}$ in die Ungleichheiten (5.) substituirt, so ergiebt sich

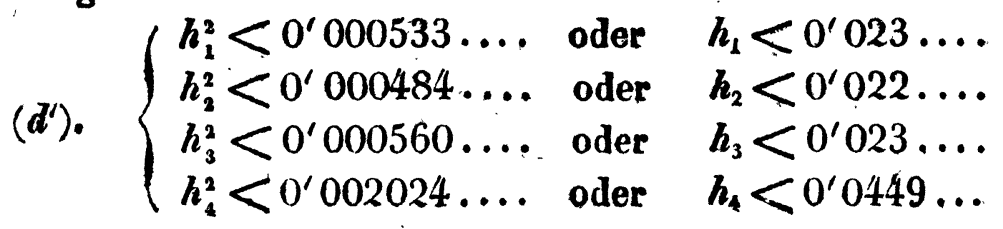


Diese Grenzwerthe von $h_{1}$ und $h_{\mathbf{2}}$ finden in der That, vermöge der Ungleichheiten $\left(c^{\prime} \cdot\right)$, Statt. Hingegen ist erst zu zeigen nöthig, dafs die Grenzwerthe von $h_{3}$ und $h_{4}$ ebenfalls noch bestehen; denn die Ungleichhei-

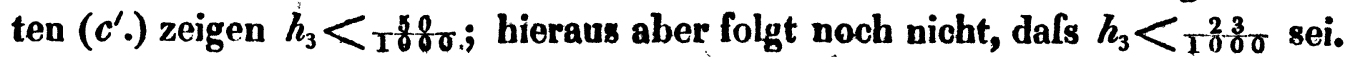
Ein Gleiches gilt von $h_{4}$. Um über diesen Punct ins Klare zu kommen, untersuche mau etwas näher jene Wurzeln der Gleichung in $y$, die zwischen 4 und 5 und zwischen 2 und 3 liegen:

Da die erste dieser Wurzeln zwisohen 4 und 5 liegt, so substituire man im Ausdrucke linker Hand vom Gleiohheitszeichen $y=4, y=4 \frac{1}{2}$, $y=5$. Die Resultate dieser Substitutionen bekommen in gleicher Ordnung folgende Zeichen: - , , +. Daher liegt die Wurzel zwischen $4 \frac{1}{2}$ und 5. Es hat mithin die Gleichung $\left(a^{\prime}\right.$.) eine zwischen $\frac{z}{q}$ und enthaltene Wurzel, oder die Grenzen dieser Wurzel sind

$$
0^{\prime} 222 \ldots, \text { und } 0^{\prime} 2 \text {. }
$$

Es differirt also unsere Annahme $a_{3}=1_{1}^{2} \sigma$ um eine Größse, die kleiner als $0^{\prime} 022, \ldots$ ist, oder man kann in den Ungleichheiten" $\left(\sigma^{\prime}\right.$.)

$$
h_{3}<{ }_{102300}^{2}
$$

voraussetzen; woraus die dritte der Ungleichbeiten $\left(d^{\prime}\right.$.) gereohtfertiget erscheint.

Auf gleiche Weise habe ich mich überzeugt, dafs die Annahme $a_{4}={ }_{T^{4}}^{4}$ einen Fehler $h_{4}$ hervorbringt, der kleiner als $0^{\prime} 0444 \ldots$. ist; wodurch die Existenz der vierten dieser Ungleichbeiten dargethan wird.

Nunmehr können wir zur Bestimmung von $v_{0}, v_{1}, v_{2}, v_{3}, v_{4}$ aus den Gleichungen (6.) übergehen, Wir baben bei Gelegenheit der Ausmittelung der Ungleichbeiten $\left(d^{\prime}\right)$ folgende Resultate erhalten;

Ferner ist

$$
\begin{array}{ll}
\varphi_{5}\left(a_{1}\right)=15705, & \emptyset_{5}\left(a_{2}\right)=-14110, \\
\varphi_{5}\left(a_{3}\right)=5531, & \Phi_{5}\left(a_{4}\right)=-285 .
\end{array}
$$

$$
\varphi_{6}(0)=0, \quad \varphi_{5}(1)=0 .
$$

Wenn daher, wie im vorhergehenden Beispiele, $\varepsilon_{m}=\frac{1}{10^{7}}$ angenommen und erwogen wird, dafs vermöge der hier angegebenen Werthe von $\varphi_{5}\left(a_{1}\right)$; $\varphi_{5}\left(a_{2}\right), \ldots$ die Gröfse $v_{1}$ die numerisch kleinste sein mufs, um deren Kenntnifs einzig und allein es zu thun ist, so kann man die Grö́sen $v_{11}, v_{2}$, $v_{3}, v_{4}$ unbeachtet lassen und blofs aus der zweiten der Gleichungen (6.) Crollo's Journal d. M. Bd. XVIII. Hft. 1. 
diese kleinste Gröfse $v_{1}$ bestimmen. Man findet

Daher nach (7.)

$$
v_{i}=006829 \ldots
$$

$$
\omega=v_{1}\left(1-\frac{1}{6}\right)=0^{\prime} 05690 \ldots
$$

Setzen wir in (I.) $w=\frac{5}{100}=\frac{1}{20}$, so hat man

$$
\begin{aligned}
\int_{0}^{1} e^{-\left(x+\frac{1}{x}\right)} d x= & {\left[e^{-\left(\omega+\frac{1}{\omega}\right)}+e^{-\left(2 \omega+\frac{1}{2 \omega}\right)}+\cdots+e^{-\left(19 \omega+\frac{1}{19 \omega}\right)}+\frac{1}{2} e^{-2}\right] } \\
& -Y_{2} \phi_{1}(1) \omega^{2}+Y_{4} \phi_{3}(1) \omega^{4}-Y_{6} \phi_{5}(1) \omega^{6}
\end{aligned}
$$

Berechnet man die in der ersten Zeile innerhalb der Klammern enthaltenen Glieder mit 7 Deoimalstellen, so findet sich:

$$
\begin{aligned}
& e^{-\left(\omega+\frac{1}{\omega}\right)}=0^{r} 0000000 ; \quad e^{-\left(11 \omega+\frac{1}{11 \omega}\right)}=0^{\prime} 0936508 \text {, } \\
& e^{-\left(2 \omega+\frac{1}{2 \omega}\right)}=0^{\prime} 0000411, \quad . \quad e^{-\left(32 \omega+\frac{1}{12 \cdot \omega}\right)}=0^{\prime} 1036571 \text {, } \\
& e^{-\left(3 \omega+\frac{1}{3 \omega}\right)}=0^{\prime} 0010954, \quad e^{-\left(13 \omega+\frac{1}{13 \omega}\right)}=0^{\prime} 1120891 \text {, } \\
& e^{-\left(4 \omega+\frac{1}{4 \omega}\right)}=0^{\prime} 0055166, \quad e^{-\left(14 \omega+\frac{1}{14 \omega}\right)}=0^{\prime} 11900 \% 2 \text {, } \\
& e^{-\left(5 \omega:+\frac{1}{5 \omega}\right)}=0^{\prime} 0142642, \quad e^{-\left(15 \omega+1 \frac{1}{15 \omega}\right)}=0^{\prime} 1245145 \text {, } \\
& e^{-\left(6 \omega+\frac{1}{6 \omega}\right)}=\theta^{\prime} 0264279 ;, \quad e^{-\left(16 \omega+\frac{1}{16 \omega}\right)}=0^{\prime} 1287350 \text {, } \\
& e^{-\left(i \omega+\frac{P}{7 \omega}\right)}=0^{\prime} 0404721, \quad e^{-\left(17 \omega+\frac{1}{17 \omega}\right)}=0^{\prime} 1318000 \text {, } \\
& e^{-\left(8 \omega+\frac{1}{8 \omega}\right)}=0^{\prime} 0550232 ; \quad e^{-\left(18 \omega+\frac{1}{18 \omega}\right)}=0^{\prime} 1338400 \text {, } \\
& e^{-\left(3 \omega+\frac{1}{3, \omega}\right)}=0^{\prime} 0690985, \quad \therefore \quad e^{-\left(19 \omega+\frac{1}{19 \omega}\right)}=0^{\prime} 1349796 \text {, } \\
& e^{-\left(10 \omega+\frac{1}{10 \omega}\right)}=0^{\prime} 0820850, \quad \quad \frac{1}{2} e^{-\left(20 \omega+\frac{1}{20 \omega}\right)}=0^{\prime} 06766 \% 6 \text {. }
\end{aligned}
$$

Addirt man diese Zahlen-Ausdrücke und multiplicirt die Summe mit $\omega=\frac{1}{20}$, so erhält mans

$\theta^{\prime} 072198245$.

Ferner hat man wegen $\phi_{1}(1)=0, \phi_{5}(1)=0$ blels das Glied: $+Y_{4} \varphi_{3}(1) w^{4}$ zu berechnen und zur eben gefundenen Zabil binzuzufügen. Dieser Zablenwerth ist :

$$
\text { 0'000000007048 ... }
$$


4. Raabe, zur Berechnung bestimmter Integrale.

Es beginnt also der Einflufs der Correctionsreihe erst in der neunten Decimalstelle und man hat

$$
\int_{0}^{1} e^{-\left(x+\frac{1}{x}\right)} d x=0^{\prime} 07219825 \ldots
$$

Wird die siebente Decimalstelle durch die achte corrigirt, so hat man

$$
\int_{0}^{1} e^{-\left(x+\frac{1}{x}\right)} d x=0^{\prime} 0721983
$$

Der bei diesem Resultate mögliche Fehler ist kleiner als $\varepsilon_{m}=\frac{1}{10^{7}}$. Aus der letzten Gleichung folgt auch die folgende:

$$
\int_{0}^{\infty} e^{-\left(x+\frac{1}{x}\right)} d x=0^{\prime} 2797318
$$

welche denselben Genauigkeitsgrad besitzt.

Zürich, im October 1836. 